\title{
La science-fiction, imaginaire des ingénieurs et des innovateurs.
}

\author{
Science fiction, imaginary of engineers and innovators.
}

\author{
Thomas Michaud ${ }^{1}$ \\ ${ }^{1}$ Chercheur indépendant, France, michaud.thomas@yahoo.fr
}

\begin{abstract}
RÉSUMÉ. La science-fiction est un imaginaire technique de plus en plus influent dans les laboratoires et centres de R\&D. Les ingénieurs et scientifiques développent des projets qui semblent réaliser les histoires de science-fiction les plus futuristes. L'innovation et la créativité sont influencées par un imaginaire technique de plus en plus formaté pour correspondre aux exigence des entreprises souhaitant proposer aux consommateurs des produits rêvés et imaginés par des auteurs de science-fiction. Quelle est l'influence de cet imaginaire sur les ingénieurs, dans leur formation et dans leur activité professionnelle ? Quels sont les secteurs les plus touchés par cette influence ? Faut-il considérer la sciencefiction et le design fiction comme des pratiques utiles ou positives ou au contraire comme des dangers pour l'éthique de la $R \& D$ ?
\end{abstract}

ABSTRACT. Science fiction is an increasingly influential technical imaginary in laboratories and R\&D centers. Engineers and scientists develop projects that seem to realize the most futuristic science fiction stories. Innovation and creativity are influenced by a technical imaginary, increasingly formatted to meet the requirements of companies wishing to offer consumers products dreamed up and imagined by science fiction writers. What is the influence of this imaginary on engineers, their training and their professional activity? Which sectors are most affected by this influence? Should we consider science fiction and design fiction as useful and positive practices or, on the contrary, as dangers for the ethics of R\&D?

MOTS-CLÉS. Science-fiction, imaginaire, innovation, design fiction, créativité.

KEYWORDS. Science fiction, imaginary, innovation, design fiction, creativity.

La science-fiction est un type d'imaginaire particulièrement populaire dans les discours des innovateurs et des entrepreneurs, dans de nombreux secteurs comme les technologies convergentes ou l'industrie spatiale. Les organisations doivent s'adapter à des imaginaires en permanente évolution, utopiques ou dystopiques, mais aussi très influents dans la définition des stratégies et des objectifs de la Recherche et Développement (R\&D). L'imaginaire science-fictionnel est particulièrement influent dans les territoires les plus innovants. Les Etats-Unis et le Japon proposent régulièrement des fictions qui fascinent un nombre considérable d'individus et d'esprits passionnés par les techno-sciences. Un pacte imaginaire unifie les visions du futur d'une communauté d'ingénieurs et de scientifiques internationale à l'origine des innovations qui transforment radicalement les modes de vie.

De quelle manière les ingénieurs sont-ils influencés par l'imaginaire, et particulièrement par la science-fiction? La rationalité scientifique est-elle compatible avec cette culture, l'imaginaire étant parfois dénoncé comme un dérivatif néfaste à la raison ? De quelle manière les organisations gèrentelles la science-fiction, par exemple dans les grands groupes de télécommunications, de nanotechnologies, de biotechnologies, mais aussi dans l'aéronautique, l'aérospatiale, et bien d'autres secteurs? Le nombre de cas d'organisations, entreprises et institutions ayant recours à la sciencefiction à des fins de prospective grandit chaque jour. Quelles sont les œuvres qui jouent ou ont joué un rôle important dans l'imaginaire des ingénieurs et scientifiques? Selon quels mécanismes psychologiques et sociologiques?

Le Design Fiction et le Science Fiction Prototyping sont des exemples de pratiques qui utilisent la science-fiction pour stimuler la créativité et inventer des technologies utopiques initiatrices de processus d'innovation. Le terme Design Fiction fut inventé par l'auteur de science-fiction Bruce Sterling en 2005. Il est depuis devenu un concept adopté par de plus en plus d'acteurs économiques. 
Les spécialistes de cette pratique apportent des conseils aux entreprises, en proposant diverses approches, comme l'invention de fictions technologiques permettant de détecter les concepts prometteurs et innovants. La culture science-fictionnelle est commune à un grand nombre d'acteurs, et notamment chez les ingénieurs, catégorie professionnelle qui nous intéresse ici particulièrement. La sociologie des sciences et des organisations, ainsi que la sociologie de l'imaginaire ont apporté ces dernières années des connaissances sur les interactions entre la fiction, la technologie, et la R\&D. Ce numéro permettra d'aborder un grand nombre de théories sur l'apport de la science-fiction à l'imaginaire des ingénieurs et à l'innovation technoscientifique.

L'article de Marianne Chouteau et Céline Nguyen interroge la fonction de la science-fiction dans l'élaboration de la culture technique. Celle-ci est présentée comme indispensable pour enrayer d'éventuelles dérives d'une société de plus en plus dominée par des technologies parfois effrayantes en raison de leur potentielle dangerosité pour le genre humain. Les deux auteurs font part de leur expérience d'enseignantes dans une école d'ingénieurs, où elles proposent différentes approches aux étudiants faisant appel à leur imaginaire. Ces derniers sont souvent influencés par une science-fiction consultée sous la forme de romans, de films ou de séries. Ces récits contribuent notamment à stimuler une réflexion éthique sur l'insertion des innovations dans la société. Ainsi, la science-fiction est au service de la culture technique. Les deux auteurs ont réalisé il y a quelques années une recherche ${ }^{1}$ particulièrement éclairante sur les usages de la science-fiction dans une école d'ingénieurs, et cet article propose son enrichissement et son dépassement.

L'article de Jean-Louis Ermine et Pierre Saulais est particulièrement intéressant car il propose une étude approfondie sur un auteur de science-fiction française important, Richard Bessière. Les auteurs rappellent quelques informations sur le contexte éditorial dans années 1950, et la production abondante de l'écrivain, qui fut le premier et plus prolifique auteur de la collection Anticipation, avec la publication de 98 livres de 1951 à 1985. Devant cette production considérable, 4 titres sont sélectionnés pour faire l'objet d'une analyse sur les rapports entre la science-fiction, l'anticipation, et la R\&D. Dans Objectif Soleil (1956), Réaction déluge (1959), Terre degré O (1960), le thème du réchauffement climatique et de l'effet de serre est abordé. Dans Plus égale moins (1961), c'est le sujet des conséquences pour l'économie et les sociétés humaines de l'invention d'une machine capable de produire des objets à très bas prix qui est traité. L'avènement d'une société d'abondance est aussi une perspective promise par les inventeurs et adeptes de l'impression 3D depuis les années 2010. Après une présentation des enjeux scientifiques et politiques du réchauffement climatique et de l'impression 3D, les auteurs se demandent si la fiction anticipative est en capacité d'influencer en profondeur l'activité de R\&D. Ils expliquent notamment que «si la fiction anticipative ne nous semble pas en capacité d'influencer en profondeur l'activité de Recherche \& Développement, nous lui reconnaissons la capacité de contribuer à agir positivement sur l'état créatif ». L'intérêt de cet article est de se focaliser sur un écrivain important. Les auteurs évitent de surévaluer l'influence de la science-fiction sur la sphère technoscientifique, tout en relevant par exemple de nombreuses technologies imaginaires novatrices dans l'œuvre de Richard Bessière. Les lecteurs pourront approfondir leurs connaissances sur le sujet en ce référant au livre d'un des auteurs de l'article, J-L. Ermine, Météore !, l'univers fascinant de Richard Bessière (2016).

Dans un article intitulé «Des innovations technologiques au monde post-apocalyptique. Enseignements tirés des films de science-fiction », Nadine Boudou s'intéresse à la fonction des films de science-fiction dans lesquels le progrès techniques a mené à des situations catastrophiques. Le genre post-apocalyptique est d'ailleurs de plus en plus influent dans l'imaginaire technique. L'auteur s'intéresse aussi à la série Real Humans, qui montre les dangers d'une commercialisation massive de

\footnotetext{
${ }^{1}$ CHOUTEAU M., FAUCHEUX M., NGUYEN C., «Les élèves ingénieurs à l'épreuve de la fiction. Quelles relations entretiennentils avec les mondes scientifiques et techniques fictionnels ? », Les Enjeux de l'Information et de la Communication, n 16/3A, 2015, p. 69 à 82 [en ligne] URL : http://lesenjeux.u-grenoble3.fr/2015-supplementA/22-Chouteau-Faucheux-Nguyen/index.html.
} 
robots humanoïdes dans la société. L'imaginaire dévoile aussi les peurs de l'avenir d'un auteur ou d'une société. La science-fiction contemporaine révèlerait ainsi les limites d'un progrès technique qui pourrait mener à la destruction de l'Humanité, voire de la planète. Les films post-apocalyptiques soulignent la fragilité d'une civilisation technologique qui se conçoit pourtant toute puissante. L'auteur s'intéresse aussi aux thèmes développés dans la climate fiction, particulièrement abondante depuis que le thème du réchauffement climatique alerte sur les dangers d'un progrès technique incontrôlé. Nadine Boudou explique que «L'hypothèse de la fin d'un monde sur laquelle ces scénarios s'appuient peut être utile aux innovateurs et les inspirer dans leurs recherches afin que celles-ci soient mises au service du bien commun ». La science-fiction est en effet une puissante critique de l'impact des technosciences sur les écosystèmes et sur les sociétés humaines. Le recours à la philosophie est utile, car elle permet d'instaurer un dialogue vertueux entre les ingénieurs et innovateurs, et le reste d'une Humanité inquiète de son devenir.

Florin Alexa-Morcov propose un texte intitulé «Ingénieurs contre designers. Transposition de l'imaginaire technique dans le visuel - «une vérité science-fiction»». Il rappelle les conditions d'émergence des designers, garants de l'esthétique des produits, et leurs relations avec les ingénieurs, à la rationalité spécifique. La recherche du nouveau est considérée comme un moteur important de l'innovation. Pour rendre un produit désirable, les ingénieurs et les designers doivent toutefois travailler de concert. A travers plusieurs exemples de produits à succès, Florin Alexa-Morcov montre que «les nouveaux objets et leurs représentations, présentées comme une conséquence du processus d'innovation, dans le cadre des « utopies performatives » sont conçus pour faire connaître aux consommateurs, au moins en partie, la dimension cachée du processus d'innovation ». Les designers sont présentés comme des magiciens qui finissent par générer un système d'objets source de désirs guidés par une dimension fictionnelle souvent cachées ou imperceptible.

Nicolas Minvielle, Remy Hemez et Olivier Wathelet, spécialistes du design fiction exposent une études des représentations des fantassins dans plus de 300 œuvres de fiction. Ils s'interrogent sur la fonction de ces récits dans le secteur militaire. Si l'imaginaire permet de se projeter, il est aussi influencé par les spécialistes de certains secteurs comme l'armée. Le design fiction devient depuis quelques années une méthode de stimulation de la créativité dans les services de stratégie des institutions et même de certaines entreprises. Par ailleurs, les auteurs et réalisateurs de romans et de films de science-fiction ont recours à des experts afin de proposer des représentations réalistes des technologies du futur. L'industrie hollywoodienne est ainsi au service d'un storytelling particulièrement utile aux ingénieurs et designers pour inventer les innovations de demain. Les auteurs proposent aussi une réflexion sur la méthodologie à mettre en place pour étudier et cartographier des imaginaires ayant la capacité de « constituer un laboratoire pour projeter des visions d'avenir crédibles et pertinentes ».

Enfin, l'article «L'apport de la science-fiction aux discours technoscientifiques sur la conquête de Mars » se concentre sur l'imaginaire martien. Le secteur spatial est très influencé par une sciencefiction qui puise aussi une grande part de son inspiration dans les découvertes scientifiques des grandes agences comme la NASA ou l'ESA. L'imaginaire de différents acteurs de l'association Planète Mars, fondée par l'ingénieur américain Robert Zubrin, ainsi que l'influence de la science-fiction sur le discours de l'entrepreneur Elon Musk, seront abordés. Le projet de colonisation de Mars mobilise de plus en plus d'acteurs d'un secteur spatial en pleine mutation, le rôle des grandes agences étant relativisé au profit d'un secteur privé de plus en plus conscient des perspectives économiques de l'espace. Si la conquête de Mars fut un sujet longtemps cantonné à des communautés d'ingénieurs et de fans de science-fiction, ce projet imaginaire pourrait bien muter en une idéologie technopolitique nommée marsisme. 